\title{
Application of Empirical Wavelet Transform in Digital Image Watermarking
}

\author{
Mohammad Keivani ${ }^{1}$, Amir Mahdi Sazdar ${ }^{2}$, Jalil Mazloum²*, Akram Esvand Rahmani ${ }^{3}$ \\ ${ }^{1}$ Department of Electrical \& Electronic Engineering, Faculty of Engineering, Islamic Azad University of Arak, Arak \\ 3836119131, Iran \\ ${ }^{2}$ Department of Electrical Engineering, Shahid Sattari Aeronautical University of Science and Technology, Tehran \\ 1384663113, Iran \\ ${ }^{3}$ Department of Computer Software Engineering, Arak University, Arak 38481-77584, Iran
}

Corresponding Author Email: jalil.mazloum@ssau.ac.ir

https://doi.org/10.18280/ts.370517

Received: 13 March 2020

Accepted: 20 September 2020

\section{Keywords:}

digital watermarking, empirical wavelet transform, copyright, alpha blending

\begin{abstract}
Nowadays, rapid growth of the Internet and digital multimedia technologies make it possible to duplicate data without any loss of quality and at a very low cost. In this regard, manipulation of documents will easily be accomplished by applying digital art and without the copyright owner's permission. To deal with situation, more diverse security requirements are introduced every day. Watermarking is considered as one of the methods used for achieving this purpose. Watermarking is focused on inserting a subtle signal between the host media data somehow it does not change the original data, but they can be extracted if necessary. They are also used as a claim for ownership of the digital effect.

Various methods have been presented for watermarking so far. These methods have been developed to overcome the weaknesses of previous methods. Empirical wavelet transform is taken into account as a new method for hiding and extracting military digital images with respect to alpha composition. As such, it is resistant to noise, low pass filter and compression. Analytical studies showed that this method is more efficient than other methods from quantitative and qualitative point of view.
\end{abstract}

\section{INTRODUCTION}

Throughout history and since humans have been able to communicate with each other, the ability to communicate in a secret way has been taken into consideration as an important demand. In the meantime, communication security is considered as a key need, and it is becoming more and more necessary every day [1]. In these ways, a watermark can be either digital image or data that is stored within a digital content. Watermarking is only important in digital watermarking methods [2]. But in some specific applications (e.g. military imaging devices), both watermarking and image retrieval are very important. For example, in capturing satellite imagery used in military applications, in addition to secret information, it is essential to provide an accurate picture for enemy positions and equipment. To achieve the purpose, the original image must be retrieved after extracting watermarking. In this case, the image is equivalent to the host image. So digital watermarking can be seen as an emerging technique to protect intellectual property and copyright. Watermarking is made by incorporation of secondary data and information within digital content, so that human beings cannot easily comprehend it, but they are diagnosed by computer algorithms [3]. Digital watermarking method is proposed for embedding and extraction of copyright protection based on discrete wavelet transform (DWT) and discrete cosine transform (DCT) [4]. The two methods take benefit from the advantages of both techniques and make one hybrid method. The combined method is applied in two-dimensional images (i.e. original cover image and watermark image). This watermarking method provides good performance and strong robustness. A method is developed based on 1-level discrete wavelet transform to insert and extract the watermark in original image by using alpha blending [1]. This technique is much simpler and robust than others. Ingale and Dhote [5] proposed an image watermarking method based on fast DCT algorithm to implement it in digital signal processor. The fast DCT algorithm has been considered to reduce the complexity of two-dimensional image transformation. In this regard, embedding/decoding an image watermark can be completed in real time within 0.33 seconds. The quality of both watermarked image and extracted (retrieved) watermark remains excellent. It is shown that the watermarking method is an efficient and robust technique for data cropping, transmission loss and compression/decompression.

Wavelet transform is employed to develop a method for watermarking based on three levels of decomposition. As such, RGB images have been used to test this algorithm. This has been tested and examined using coefficient of $\mathrm{a}$ in the latent image [5].

An attempt is made to analyze robustness for different test images. To resist various attacks, Empirical Mode Decomposition (EMD) is used. Performance evaluation for an image watermarking includes robustness, imperceptibility, watermark capacity and security [6].

We presented a robust technique based on Bi-dimensional Empirical Mode Decomposition (BEMD). Intrinsic Mode Functions (IMFs) extracted through BEMD are watermarked with ponderation using patchwork for the first 4 BIMFs [7]. We have carried out work on greyscale images using basic 
BEMD algorithm and found that if watermarked image is attacked by noise, visibility of the recovered mark will considerably decrease. Therefore, we have proposed a technique using Ensemble Empirical Mode Decomposition (EEMD) and experiments ascertain better robustness against noise attacks.

A method is represented using discrete cosine converter for digital images. In this watermarking method, digital watermarks can effectively be embedded and extracted without presence of host image. In this method, the complexity is reduced for two-dimensional transformations. The results show that the watermarking method can be efficient and robust against transmission and compression [8].

In a recent approach [9], a new image watermarking method is proposed that uses Gaussian filters and a first-order partial differential matrix. This method uses corral conversion and strong parameters to preserve the edge.

Another method [10] uses a watermarking based on the singular value decomposition (SVD) method. In this method, which is based on a chaotic map design, the secret key is first extracted from the host image and watermark image. This key is used to generate a new disordered matrix and scalable multiple scaling factors (CMSF) to increase the sensitivity of the proposed design.

Recent researches have also suggested the Hybrid Transdomain and Particle Swarm Optimization (PSO) algorithm [11], the APBT-based algorithm and SVD [12], Spatial Domain technique, and lifting and compounding [13].

Due to the fact that other methods, such as EMD and its improved versions lack mathematical theory and are experimental. On the other hand, as methods such as DWT are based on a mathematical model but have pre-prescribed violins, we have sought to use an experimental wavelet transform (EWT) [14] method for digital cryptography, because certain decomposed components of image are processed based on their images.

It is taken into consideration as one of the advantages of empirical wavelet transform over traditional discrete wavelet transform.

Also, Watermarking algorithms are divided into two groups encompassing blind and non-blind watermarking. In the blind watermarking, no original image extraction is required, but in the non-blind watermarking, original image extraction is required [1]. In this article, we use non-blind watermarking. The article is organized as follows. Section 2 contains an introduction for experimental wavelet transform. Section 3 presents the proposed method in two parts including extraction and analysis.

\section{INTRODUCTION TO EMPIRICAL WAVELET TRANSFORM}

Until now, fixed filters were used for multimedia models in each image. This procedure makes it impossible to display the optimal filters. A better way is to produce filters based on image features.

Huang et al. [15] proposed a new method for signal analysis based on empirical mode analysis. The purpose of this method is to find oscillatory narrow band components called intrinsic state functions. The advantage of this method is associated with the issue that it does not require any prior assumptions. This method was used for watermarking process.

However, there were two major problems dealing with applying the empirical mode decomposition method. First, it lacked mathematical theory, and second, it did not guarantee that different input images would be adapted to each other. This has led to production of images that are different from input image and bring artifacts.

As we know, discrete wavelet transform is also used for image decomposition. In this way, when the image is transmitted into transform domain, the pre-prescribed design is broken down into approximations and details layers, which in turn affect the signal analysis results. However, their wavelet base sets are fixed and make it impossible to optimize the filter design for displaying image. So it is the best alternative to design a set of filters based on the processed signals. This has led to emergence of experimental wavelet transforms that do not have the above problems [7]. The experimental wavelet transform method had strong theoretical basis and was theoretically better than the empirical mode decomposition and discrete wavelet transform methods.

It is due to this has the ability to build empirical wavelet sets based on adaptive filter banks. Experimental wavelet transform is concerned with multi-resolution decomposition of a signal that decomposes the image hierarchically.

A new method called Experimental Wavelet transform method is presented [16]. This method decomposes images into approximation and details layers, which are consisted of low and high frequency information, respectively. The approximation layer retains low frequency information such as critical information from images such as intensity levels and structures. Whereas, minor layers retain high frequency information such as edges and textures.

Therefore, great performance of this method in the process of image fusion [17] motivated us to apply experimental wavelet transforms in watermarking. So far, this method has not been used for watermarking. This paper is aimed to employ this method for watermarking. In the next section, a full description is represented for two-dimensional experimental wavelet transform algorithm [18].

\subsection{Two-dimensional empirical wavelet transform}

In the real world, 2D signals need to be processed. Therefore, it is important to develop a two-dimensional empirical wavelet transform method. An alternative is to convert one-dimensional empirical wavelet transform to twodimensional empirical wavelet transform. Then, the approximation layer and empirical layer are mapped to $2 \mathrm{D}$ space. However, we may lose some spatial information of adjacent pixels in this process. For this reason, twodimensional Littlewoods-Paley wavelet transform is used among different wavelet transforms methods.

In the two-dimensional empirical wavelet transform, $\mathrm{f}_{2}(\mathrm{t})$ shows the two-dimensional input signal (input image) and Fourier transform of $\mathrm{f}_{2}(\mathrm{t})$ as $\mathfrak{F}_{t}\left(f_{2}\right)(\omega)$. Therefore, the approximation layer and details layers of Eq. (1) and Eq. (2) can be obtained [16]:

$$
\begin{aligned}
& \mathrm{W}\left(f_{2}\right)(0, \mathrm{t})=\mathfrak{F}_{\omega}^{-1}\left(\mathfrak{F}_{t}\left(f_{2}\right)(\omega) \overline{\mathfrak{F}_{t}\left(\phi_{1}\right)(\omega)}\right) \\
& \mathrm{W}\left(f_{2}\right)(\mathrm{m}, \mathrm{t})=\mathfrak{F}_{\omega}^{-1}\left(\mathfrak{F}_{t}\left(f_{2}\right)(\omega) \overline{\mathfrak{F}_{t}\left(\varphi_{\mathrm{m}}\right)(\omega)}\right)
\end{aligned}
$$

The inverse Fourier transform with $\mathfrak{F}_{\omega}^{-1}$ is shown in the above equations. As can be seen, the approximation layer and details layers in Eq. (1) and Eq. (2) are obtained by internal multiplication of the Fourier transform of the image in 
conjugate with the decomposed layer of approximation and details layers [17].

Finally, inverse of the two-dimensional empirical wavelet transform is shown in Eq. (3):

$$
f_{2}(\mathrm{t})=\mathrm{W}\left(f_{2}\right)(0, \mathrm{t}) \diamond\left(\phi_{\mathrm{t}}\right)+\sum_{\mathrm{m}=1}^{\mathrm{M}-1} \mathrm{~W}\left(f_{2}\right)(\mathrm{m}, \mathrm{t}) \diamond \varphi_{\mathrm{m}}(\mathrm{t})
$$

In the Eq. (3), sign $\diamond$ is related to the convolution operator. In other words, integer multiplication of the input Fourier transform is approximated for the input images through sum of the Fourier transform of the input images with respect to internal multiplication of the details layers. Therefore, the twodimensional empirical wavelet transform algorithm has been introduced. Next section revolves around watermarking methods.

\section{METHODOLOGY}

The purpose of this study is to hide personal data within the cover image. To achieve this goal, a new method is developed and it is called empirical wavelet transform method. There are various methods for wavelet transform in empirical wavelet transforms that use Littlewoods-Paley 2D wavelet transform for watermarking. Also, wavelet watermarking is chosen because it reduces the risk of any distortion such as low pass filters that modify high frequency components, but destroys attacks (e.g. crop) destroy the entire watermarked image, and thus it does not resist.

\subsection{Watermark embedding using EWT}

First, the cover image will be received. In the second step, it is divided into four components including low-frequency approximation component, high-frequency components, vertically, horizontally and diagonally. In the next step, the hidden image is covered inside another image using alpha blending method.

Finally, coefficients of the hidden image are hidden within the coefficient of approximation of the cover image. It is due to the coefficients of approximation contain more information than watermarking images. In this way, watermarking is performed in such a way that it is naturally seen in the images.

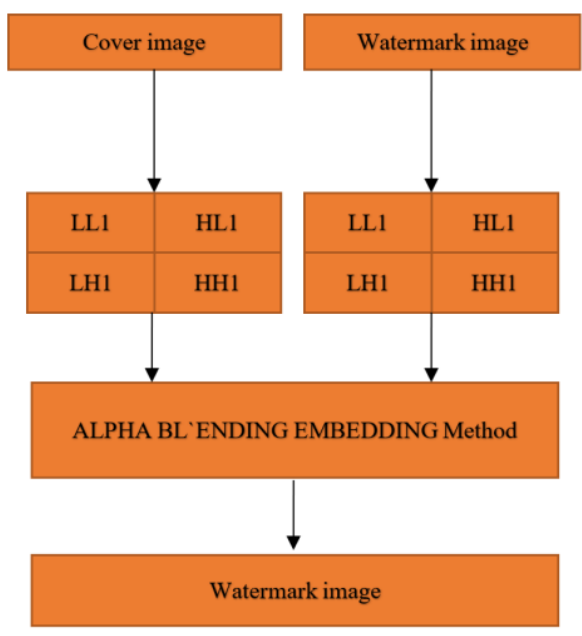

Figure 1. Watermark embedding method

\subsubsection{Alpha blending method}

Alpha blending is involved in process of blending an image with a background to create a crisp, distinctive look [1]. Eq. (4) expresses the alpha blending formula used for watermarking.

Watermarked image $=\mathrm{A} \times(\mathrm{LL} 1)+\mathrm{B} \times(\mathrm{WM} 1)$

$\mathrm{A}, \mathrm{B}=$ scaling factors for cover and watermark image, respectively.

LL1 $=$ low frequency approximation of cover image

$\mathrm{WM} 1=$ watermark image

Then, inverse discrete transform is applied to watermarked image coefficient to generate secure watermarked image. The general way to conceal is to hide the image inside the cover image as shown in Figure 1.

\subsection{Extraction of the watermarked image using EWT}

Both watermarked image and cover image are decomposed into their sub bands by applying EWT to both images. Now we use cover image to extract the watermark image using nonblind watermarking method. Then, alpha blending formula is applied to recover the watermark image from watermarked image.

Here, the approximate sub band frequency layer of the cover image is multiplied by particular scaling factor, and then it is subtracted from watermarked image coefficient.

\subsubsection{Alpha blending}

The alpha blending formula used for watermark extraction is:

Recovered watermark $=\left(\mathrm{WM}-\mathrm{A}^{*} \mathrm{LL} 1\right)$

$\mathrm{WM}=$ watermarked image

$\mathrm{A}=$ scaling factor for cover image

LL1 = low frequency approximation component of cover image

To generate the final watermark extracted image, inverse empirical wavelet transform is applied to watermark image coefficient. The general method is to extract the hidden image from the cover image as shown in Figure 2.

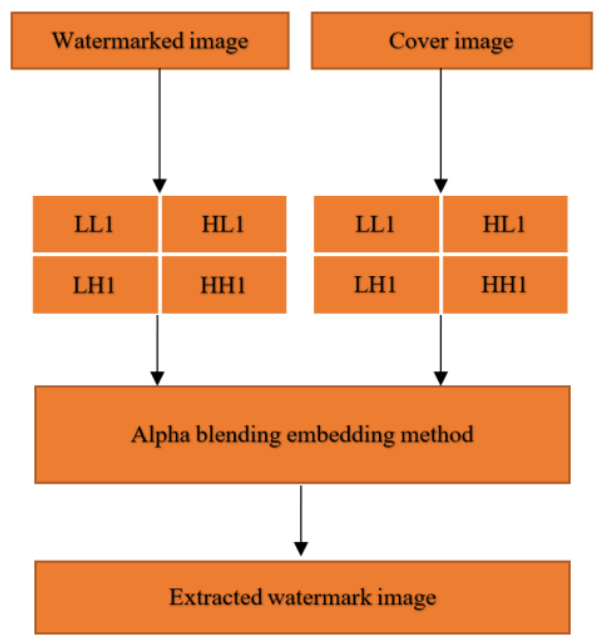

Figure 2. Watermark extraction method

\section{RESULTS AND DISCUSSION}

To implement and test the proposed method, a computer 
system was used with the following specifications: Windows 8, 64-bit, Intel Core i7-4720 CPU@2.60 GHz, 6 GB memory. MATLAB R2019a software package was used for analysis process. To compare the robustness of the proposed method with the other methods, PSNR (Peak Signal to Noise Ratio) and NCC (Normalized Cross Correlation) criteria are used as shown in Eq. (5) and (6).

$$
\mathrm{PSNR}=10 \times \log _{10} \frac{255^{2}}{\sum_{i=1}^{m} \sum_{j=1}^{n}\left(P_{i j}-q_{i j}\right)^{2}}(\mathrm{db})
$$

where, $P_{i j}$ represents the values for pixels in rows $i$ and $j$ of the host image, and $P_{i j}$ denotes the pixel values in rows $\mathrm{i}$ and $\mathrm{j}$ of the embedded image.

$$
\mathrm{NCC}=\frac{\sum_{i=1}^{m} \sum_{j=1}^{n} P_{i j} q_{i j}}{\sum_{i=1}^{m} \sum_{j=1}^{n} P_{i j}{ }^{2}}
$$

where, $P_{i j}$ represents the values for pixels in rows $i$ and $j$ of the host image, and $P_{i j}$ denotes the pixel values in rows $i$ and $j$ of the embedded image.

Two color image pairs are used to perform the watermarking process. In the first pair of images, the first color image is related to the image of a pepper and it is used as cover image (Figure 3). The second color image is relied on the image of an air rocket used as a cover image (Figure 4). This type of watermarking is the hidden purpose of military equipment. Low frequency content is considered for both images. As mentioned, the alpha compound is used. The size of both images is 256 by 256 . The watermarking image lies within the cover image, and the value of $A$ varies from 0.1 to 0.9 .

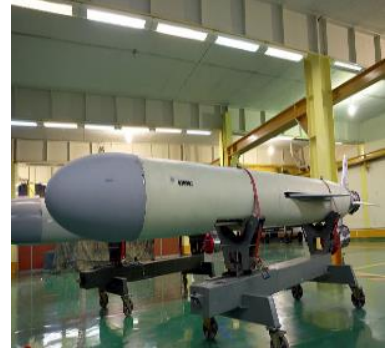

(a) hidden image

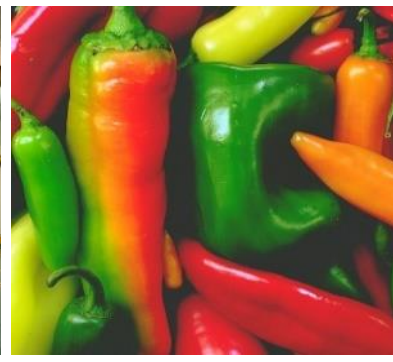

(b) Cover image
Figure 3. Input images

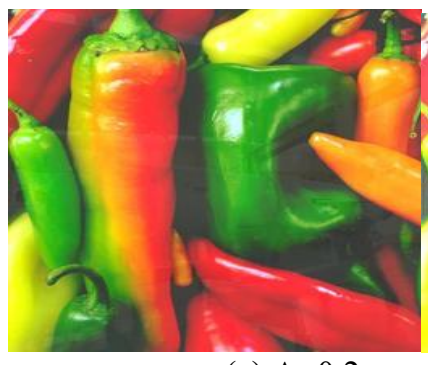

(a) $\mathrm{A}=0.2$

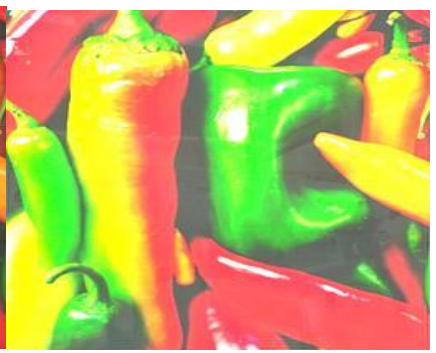

(b) $\mathrm{A}=1$
Figure 4. Hidden images

The value of $\mathrm{B}$ is always constant. The best result is obtained when $\mathrm{A}=0.2$, which makes the watermarking image process more clear and complete in the watermarking process. For the watermarking image extract process, the value of $\mathrm{A}$ varies from 0.9 to 0.1 , depending on the watermarking algorithm. If A decreases to 0.2, the hidden image becomes darker and is completely invisible. In Figure 4, sections A and $\mathrm{B}$ show the hidden images with different values for A. Figure $5 \mathrm{a}$ and $\mathrm{b}$ show the recovery of latent images based on different values for $\mathrm{A}$.

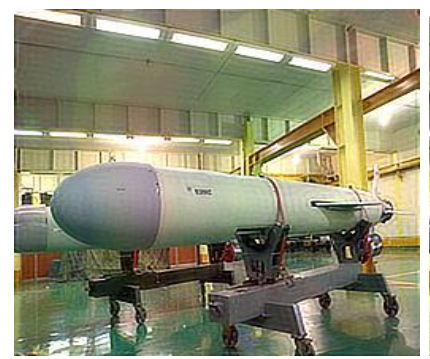

(a) $\mathrm{A}=0.2$

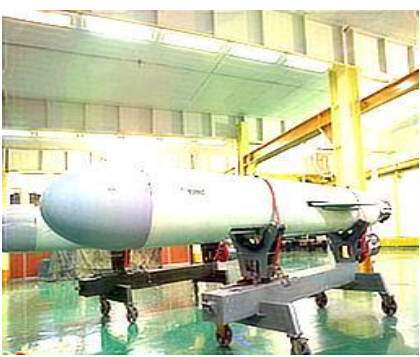

(b) $\mathrm{A}=0.95$
Figure 5. Extract watermarking images

\subsection{Extraction of hidden images using a variety of a and b values}

In the proposed method, the results are cached and retrieved using two levels of analysis. Second level usually provides better results than first level decomposition due to more decomposition. At the second level, the approximate layer, LL1, is decomposed into four subgroups including LL2, LH2, HL2 and HH2. The results of the PSNR at the two breakdown levels are listed in Tables 1 and 2. The values obtained from the proposed method are plotted in both tables. It is done for both watermarking process and extract process.

Table 1. Image watermarking with different A and B values

\begin{tabular}{ccccc}
\hline s.no & A & B & PSNR & observation \\
\hline 1 & 0.1 & 0.1 & 55.98 & \\
2 & 0.2 & 0.1 & 53.06 & \\
3 & 0.3 & 0.1 & 51.15 & \\
4 & 0.4 & 0.1 & 59.86 & \\
5 & 0.5 & 0.1 & 57.62 & Best result \\
6 & 0.6 & 0.1 & 56.64 & \\
7 & 0.7 & 0.1 & 55.87 & \\
8 & 0.8 & 0.1 & 55.27 & \\
9 & 0.9 & 0.1 & 54.78 & \\
10 & 1 & 0.1 & 54.23 & \\
\hline
\end{tabular}

Table 2. Extract image watermarking with different A and B values

\begin{tabular}{ccccc}
\hline s.no & A & B & PSNR & observation \\
\hline 1 & 0.1 & 0.1 & 57.34 & \\
2 & 0.2 & 0.1 & 54.47 & \\
3 & 0.3 & 0.1 & 54.02 & \\
4 & 0.4 & 0.1 & 51.82 & \\
5 & 0.5 & 0.1 & 57.33 & Best result \\
6 & 0.6 & 0.1 & 56.96 & \\
7 & 0.7 & 0.1 & 56.35 & \\
8 & 0.8 & 0.1 & 55.52 & \\
9 & 0.9 & 0.1 & 55.04 & \\
10 & 1 & 0.1 & 54.81 & \\
\hline
\end{tabular}

Also, in the proposed method, considering that there is a time of decomposition and reconstruction processing, therefore, decomposition at different levels does not have such an effect on increasing the measurement criteria as the PSNR in the Figure 6. 


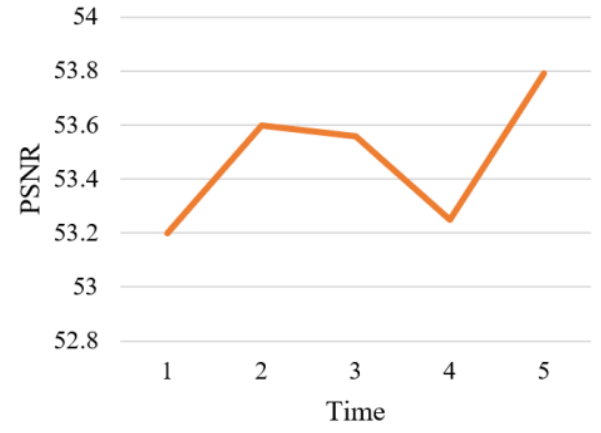

Figure 6. Relationship of the PSNR metric with time

\subsection{The robustness of the proposed method against attacks}

When images are scattered on the Internet, they can be subject to various attacks. Therefore, the watermarking method should be adopted in a way that is resistant to attacks. Finally, we will provide some examples to test the images for detection and attacks below.

\subsection{Strength to extract against white noise and salt \& pepper attacks}

In the proposed method, white noise watermarking or Gaussian noise with zero mean is added to the images. Figure 7 (a) shows the cover image after Gaussian noise attack. Figure 7 (b) depicts the watermarked image. Figure 7 (c) exhibits the extracted image after watermarking and noise removal process The quality of the extracted image and related results of the algorithm in Table 3 indicate that this method is more robust against noise attacks.

Also, Table 3 shows the effect of Correlation by applying salt and peppers noise with different densities in the proposed method.

Table 3. Dependence of watermarking on images with different values of A and B

\begin{tabular}{ccccc}
\hline s.no & $\begin{array}{c}\text { Cover image with A } \\
\text { value }\end{array}$ & $\begin{array}{c}\text { Image watermarked with B } \\
\text { value }\end{array}$ & $\begin{array}{c}\text { Correlation values (white } \\
\text { noise) }\end{array}$ & $\begin{array}{c}\text { Correlation values (salt and peppers } \\
\text { noise) }\end{array}$ \\
\hline 1 & 0.1 & & 53.20 & 52.17 \\
2 & 0.2 & 0.1 & 54.25 & 52.47 \\
3 & 0.3 & 0.1 & 55.90 & 52.31 \\
4 & 0.4 & 0.1 & 55.95 & 53.20 \\
5 & 0.5 & 0.1 & 55.98 & 52.90 \\
6 & 0.6 & 0.1 & 56.01 & 53.54 \\
7 & 0.7 & 0.1 & 56.10 & 54.25 \\
8 & 0.8 & 0.1 & 56.22 & 54.12 \\
9 & 0.9 & 0.1 & 56.40 & 54.48 \\
10 & 1 & 0.1 & 56.80 & 55.23 \\
\hline
\end{tabular}

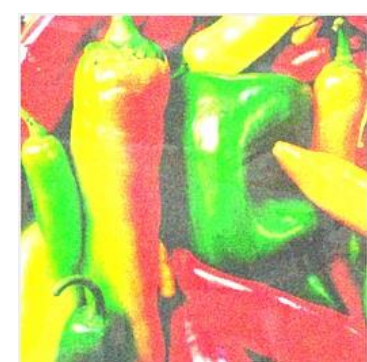

(a) The cover image after from white noise attack

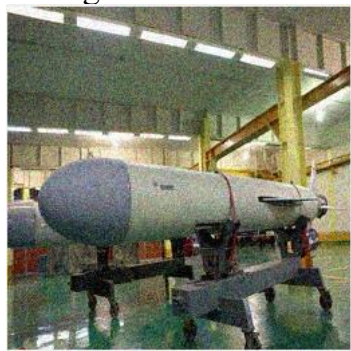

(b) The image hidden after the white noise attack

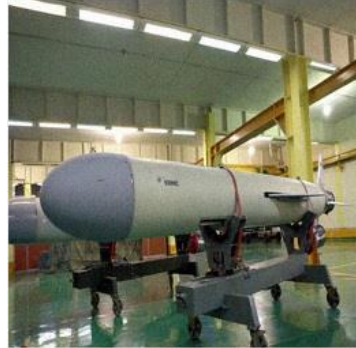

(c) The image extracted after denoising

Figure 7. The strength of the watermarking images

\subsection{Extract strength against jpeg compression}

Image compression involves in transferring important components. Thus, it tests the image watermarking versus JEPG image compression in which we paid. The compression results are shown in Table 4 . The value of coefficient indicates the quality of the compressed image. The higher the quality factor, the higher the image quality, and the lower the quality if the quality factor is lower. The values range from 0 to 100 . Dependency between the extracted image and original image is also considered for estimation of the watermarking. As such, if the correlation coefficients are closer to 1 , the consistency between the original image and extracted image is better. The results of the Table 4 demonstrate that the algorithm proposed in this paper is resistant to JEPG compression.

Table 4. Watermarking of images against compression

\begin{tabular}{c|c|c|c|c|c|c|c}
\hline JPEG quality & 20 & 40 & 50 & 70 & 80 & 90 & 100 \\
\hline Correlation & 0.5 & 0.6 & 0.7 & 0.8 & 0.92 & 0.99 & 1 \\
\hline
\end{tabular}

\subsection{Strength to extract against low pass filter}

Figure 8 (a) shows the cover image after the Gaussian lowpass filter and Figure 8 (b) displays the extracted image for comparison. Figure 8 (c) indicates the extracted image after the latent filtering process and recovery after the low pass filter.

In this section, we compare the proposed method to other techniques including DWT [5], EMD [6], and DCT [8] methods. The comparison is performed on input images of a and $b$ in Figure 3. Table 5 compares the values for PSNR and $\mathrm{NC}$ in the proposed method with other methods with respect 
to different attacks. Also, in Figures 8 and 9, we compare the proposed PNSR and NC with other methods.

Based on the results of the Table 5 and Figures 9 and 10, in the method of watermarking that performed by discrete wavelet transform, the components of the frequency bands are pre-prescribed at the time of decomposition. Thus, it is not desirable when reconstructing the image quality. In the DCT method, when parsing images, sometimes images get too much artifacts during reconstruction. It happens due to blocking, and thus it makes this algorithm less efficient.

Table 5. Comparison of the proposed algorithm with other methods

\begin{tabular}{|c|c|c|c|c|c|c|c|c|}
\hline \multirow{2}{*}{ Image type under attack } & PSNR & $\mathrm{NC}$ & PSNR & $\mathrm{NC}$ & PSNR & NC & PSNR & NC \\
\hline & \multicolumn{2}{|c|}{ EMD } & \multicolumn{2}{|c|}{ DWT } & \multicolumn{2}{|c|}{ DCT } & \multicolumn{2}{|c|}{ Proposed Method } \\
\hline Gaussian noise & 28.32 & 0.9535 & 28.97 & 0.8610 & 27.42 & 0.7549 & 55.74 & 0.9940 \\
\hline Pepper and salt noise & 29.16 & 0.9420 & 27.63 & 0.7947 & 26.99 & 0.8052 & 56.06 & 0.9867 \\
\hline Low-pass filter & 30.69 & 0.9643 & 29.14 & 0.8366 & 26.34 & 0.7825 & 56.67 & 1 \\
\hline
\end{tabular}

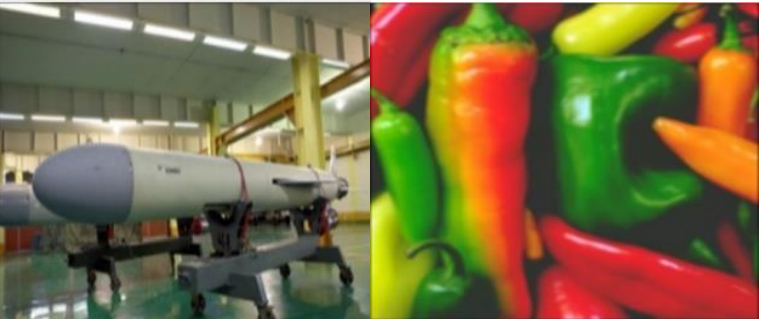

(a) Image extracted (b) Cover image

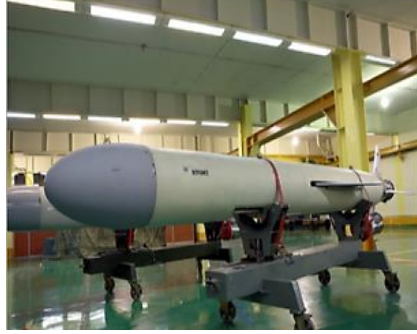

(c) Extracted image after applying low pass filter

Figure 8. Strength of images with low pass filter

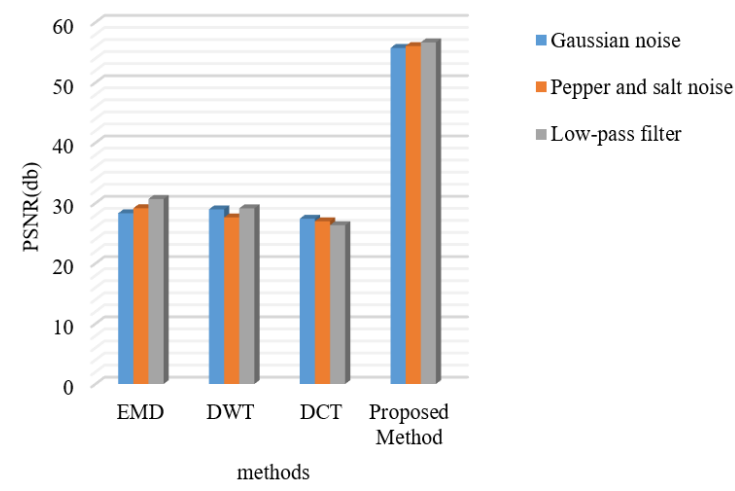

Figure 9. PSNR performance comparison

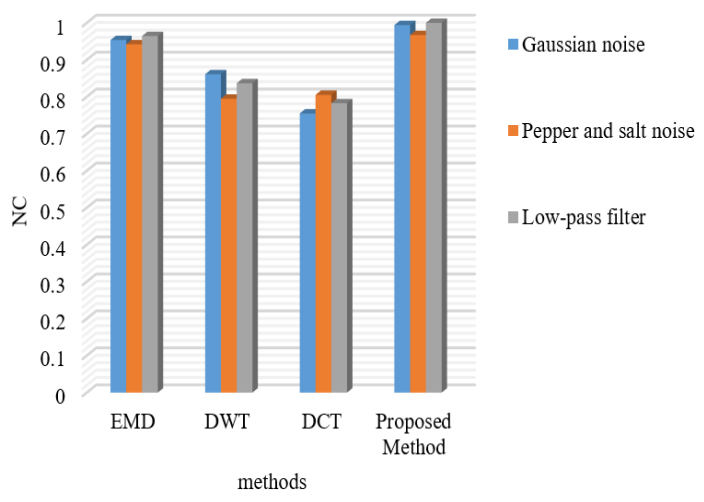

Figure 10. NC performance comparison
EMD method is considered as algorithm-based approach and lacks mathematical theory. In this method, regeneration bands may not resemble the initial state sometimes, and this issue is taken into account as one of the weaknesses of this algorithm.

Finally, the proposed method is characterized as an experimental wavelet conversion method, because the coefficients are image-based and based on mathematical theory. They are highly desirable when reconstructing the image quality and quantitatively as shown in the evaluation section. The values are higher than other methods.

The disadvantage of the experimental wavelet transform method refers to the issue that when the input images are simultaneously split into several layers, the low frequency coefficients and high frequency coefficients are considered for both images in a similar range. For this reason, it leads to exactly match the coefficients of the images at the time of reconstruction. In the same way, if the coefficients overlap when the frequency bands are overlapped, they sometimes have trouble.

\section{CONCLUSIONS}

In this paper, watermarking is presented with the aim of hiding military equipment by presenting a new method called experimental wavelet transform (EWT). In this way, the watermarking and retrieving are performed for the hidden images in a hierarchical manner. In the proposed method, the hidden image is embedded in the overlay image and retrieved using the same overlay image. The insertion and extraction of the latent image is obtained by its experimental and inverse wavelet transform and alpha composition method. Scale coefficients of A and B determine the quality of latent and retrieved images. Evaluations of the results show that performance of the proposed method is better than other traditional methods such as DWT, DCT and EMD. The proposed algorithm is also robust for recovery against common attacks such as shrinkage white noise, JPEG compression and low pass filtering. Future work can focus on other wavelet transforms such as parsing Variational Mode Decomposition (VMD) variable states for watermarking.

\section{REFERENCES}

[1] Narang, M., Vashisth, S. (2013). Digital watermarking using discrete wavelet transform. International Journal of Computer Applications, 74(20): 34-38. https://doi.org/10.5120/13029-0219

[2] Kamble, A., Agrawal, S.S. (2019). Wavelet based digital 
image watermarking algorithm using fractal images. In 2019 3rd International Conference on Electronics, Communication and Aerospace Technology (ICECA), pp. $1220-1224$ https://doi.org/10.1109/ICECA.2019.8822029

[3] Mohanarathinam, A., Kamalraj, S., Venkatesan, G.P., Ravi, R.V., Manikandababu, C.S. (2019). Digital watermarking techniques for image security: A review. Journal of Ambient Intelligence and Humanized Computing, 11: 3221-3229. https://doi.org/10.1007/s12652-019-01500-1

[4] Barnouti, N.H., Sabri, Z.S., Hameed, K.L. (2018). Digital watermarking based on DWT (discrete wavelet transform) and DCT (discrete cosine transform). International Journal of Engineering \& Technology, 7(4): 4825-4829. https://doi.org/10.14419/ijet. v7i4.25085

[5] Ingale, S.P., Dhote, C.A. (2016). Digital watermarking algorithm using DWT technique. International Journal of Computer Science and Mobile Computing, 5(5): 1-9.

[6] Kannadhasan, S., Suresh, R. (2014). EMD algorithm for robust image watermarking. Advanced Materials Research, 984-985: 1255-1260. https://doi.org/10.4028/www.scientific.net/AMR.984985.1255

[7] Shrinivasacharya, P., Kumar, K., Shankar, M.(2011). Digital image watermarking using EMD. Journal of Computing, 470-475.

[8] Tsai, S.E., Liu, K.C., Yang, S.M. (2017). An efficient image watermarking method based on fast discrete cosine transform algorithm. Mathematical Problems in Engineering, 2017: 3509258 . https://doi.org/10.1155/2017/3509258

[9] Mittal, M., Kaushik, R., Verma, A., Kaur, I., Goyal, L. M., Roy, S., Kim, T.H. (2020). Image watermarking in curvelet domain using edge surface blocks. Symmetry, 12(5): 822. https://doi.org/10.3390/sym12050822

[10] Zainol, Z., Teh, J.S., Alawida, M. (2020). A new chaotic image watermarking scheme based on SVD and IWT. IEEE Access, 8: 43391-43406.
https://doi.org/10.1109/ACCESS.2020.2978186

[11] Takore, T.T., Kumar, P.R., Devi, G.L. (2018). A new robust and imperceptible image watermarking scheme based on hybrid transform and PSO. International Journal of Intelligent Systems and Applications, 10(11): 50-63. https://doi.org/10.5815/ijisa.2018.11.06

[12] Zhang, Y., Wang, C., Wang, X., Wang, M. (2017). Feature based image watermarking algorithm using SVD and APBT for copyright protection. Future Internet, 9(2): 13-28. https://doi.org/10.3390/fi9020013

[13] Pizzolante, R., Castiglione, A., Carpentieri, B., De Santis, A., Castiglione, A. (2014). Protection of microscopy images through digital watermarking techniques. In 2014 International Conference on Intelligent Networking and Collaborative Systems, pp. 65-72. https://doi.org/10.1109/INCoS.2014.116

[14] Gilles, J. (2013). Empirical wavelet transform. IEEE Transactions on Signal Processing, 61(16): 3999-4010. https://doi.org/10.1109/TSP.2013.2265222

[15] Huang, N.E., Shen, Z., Long, S.R., Wu, M.C., Shih, H.H., Zheng, Q., Liu, H.H. (1998). The empirical mode decomposition and the Hilbert spectrum for nonlinear and non-stationary time series analysis. Proceedings of the Royal Society of London. Series A: mathematical, Physical and Engineering Sciences, 454(1971): 903-995. https://doi.org/10.1098/rspa.1998.0193

[16] Gilles, J., Tran, G., Osher, S. (2014). 2D empirical transforms. Wavelets, ridgelets, and curvelets revisited. SIAM Journal on Imaging Sciences, 7(1): 157-186. https://doi.org/10.1137/130923774

[17] Zhang, X., Li, X., Feng, Y. (2017). Image fusion based on simultaneous empirical wavelet transform. Multimedia Tools and Applications, 76(6): 8175-8193. https://doi.org/10.1007/s11042-016-3453-8

[18] Kuraparthi, S., Kollati, M., Kora, P. (2019). Robust optimized discrete wavelet transform-singular value decomposition based video watermarking. Traitement du Signal, 36(6): 565-573. https://doi.org/10.18280/ts.360612 